\title{
EULER CASE FOR A GENERAL FOURTH-ORDER DIFFERENTIAL EQUATION
}

\author{
A. S. A. AL-HAMMADI \\ Received 1 January 2004
}

\begin{abstract}
We deal with an Euler case for a general fourth-order equation and under this case, we obtain the general formula for the asymptotic form of the solutions.
\end{abstract}

2000 Mathematics Subject Classification: 34E05.

1. Introduction. In this paper, we examine the asymptotic form of a fundamental set of solutions of the fourth-order differential equation

$$
\left(p_{0} y^{\prime \prime}\right)^{\prime \prime}+\left(p_{1} y^{\prime}\right)^{\prime}+\frac{1}{2} \sum_{j=0}^{1}\left[\left\{q_{2-j} y^{(i)}\right\}^{(j+1)}+\left\{q_{2-j} y^{(j+1)}\right\}^{(j)}\right]+p_{2} y=0
$$

as $x \rightarrow \infty$, where $x$ is the independent variable and the prime denotes $d / d x$. The functions $p_{i}(x)(0 \leq i \leq 2)$ and $q_{i}(x)(i=1,2)$ are defined on an interval $[a, \infty)$, are not necessarily real-valued, and are all nowhere zero in this interval. Our aims are to identify relations between $q_{0}, q_{1}, p_{0}, p_{1}$, and $p_{2}$ that represents an Euler case for (1.1) and to obtain the asymptotic forms of four linearly independent solutions under this case. Al-Hammadi [2] obtained an asymptotic formula of Liouville-Green type for (1.1) which extends those of Walker [9]. Also in [1], we consider (1.1) with $p_{1}=q_{2}=0$ and we give a complete analysis for the case where

$$
p_{2}^{1 / 3} p_{0}=o\left(q_{1}^{4 / 3}\right) \quad(x \rightarrow \infty) .
$$

A fourth-order equation similar to (1.1) has been considered previously by Walker $[9,10]$. Eastham [4] considered an Euler case for (1.1) with $p_{1}=q_{2}=0$ and showed that this case represents a borderline between situations where all solutions have a certain exponential character as $x \rightarrow \infty$ and where only two solutions have this character. Al-Hammadi and Eastham [3] considered the case where the coefficients are small for large $x$.

The Euler case for (1.1) that has been referred to is given by

$$
\begin{aligned}
& \frac{q_{i}^{\prime}}{q_{i}} \sim \text { const } \frac{q_{1}}{q_{0}} \quad(i=1,2), \\
& \frac{\left(p_{1} q_{1}^{-1 / 2}\right)^{\prime}}{p_{1} q_{1}^{-1 / 2}} \sim \text { const } \frac{q_{1}}{p_{0}}
\end{aligned}
$$

as $x \rightarrow \infty$. 
We will use the recent asymptotic theorem of Eastham [6, Section 2] to obtain the solutions of (1.1) under the above case. The main theorem for (1.1) is given in Section 4 with some discussion in Section 5.

2. A transformation of the differential equation. We write (1.1) in the standard way [7] as a first-order system

$$
Y^{\prime}=A Y
$$

where the first component of $Y$ is $y$ and

$$
A=\left[\begin{array}{cccc}
0 & 1 & 0 & 0 \\
0 & -\frac{1}{2} q_{1} p_{0}^{-1} & p_{1}^{-1} & 0 \\
-\frac{1}{2} q_{2} & -p_{1}+\frac{1}{4} q_{1}^{2} p_{o}^{-1} & -\frac{1}{2} p_{0}^{-1} q_{1} & 1 \\
-p_{2} & -\frac{1}{2} q_{2} & 0 & 0
\end{array}\right] .
$$

As in [1], we express $A$ in its diagonal form

$$
T^{-1} A T=\Lambda
$$

and we therefore require the eigenvalues $\lambda_{j}$ and the eigenvectors $v_{j}(1 \leq j \leq 4)$ of $A$.

The characteristic equation of $A$ is given by

$$
p_{0} \lambda^{4}+q_{1} \lambda^{3}+p_{1} \lambda^{2}+q_{2} \lambda+p_{2}=0 .
$$

An eigenvector $v_{j}$ of $A$ corresponding to $\lambda_{j}$ is

$$
u_{j}=\left(1, \lambda_{j}, p_{0} \lambda_{j}^{2}+\frac{1}{2} q_{1} \lambda_{j},-\frac{1}{2} q_{2}-p_{2} \lambda_{j}^{-1}\right)^{t}
$$

where the superscript $t$ denotes the transpose. We assume at this stage that the $\lambda_{j}$ are distinct, and we define the matrix $T$ in (2.3) by

$$
T=\left(\begin{array}{llll}
u_{1} & u_{2} & u_{3} & u_{4}
\end{array}\right)
$$

Now from (2.2), we note that $E A$ is symmetric, where

$$
E=\left(\begin{array}{llll}
0 & 0 & 0 & 1 \\
0 & 0 & 1 & 0 \\
0 & 1 & 0 & 0 \\
1 & 0 & 0 & 0
\end{array}\right)
$$

Hence, be [5, Section 2(i)], the $v_{j}$ have the orthogonality property

$$
\left(E v_{k}\right)^{t} v_{j}=0 \quad(k \neq j) .
$$


We define the scalars $m_{j}(1 \leq j \leq 4)$ by

$$
m_{j}=\left(E v_{j}\right)^{t} u_{j},
$$

and the row vectors

$$
r_{j}=\left(E v_{j}\right)^{t}
$$

Hence, by [5, Section 2],

$$
\begin{gathered}
T^{-1}=\left[\begin{array}{l}
m_{1}^{-1} r_{1} \\
m_{2}^{-1} r_{2} \\
m_{3}^{-1} r_{3} \\
m_{4}^{-1} r_{4}
\end{array}\right], \\
m_{j}=4 p_{0} \lambda_{j}^{3}+3 q_{1} \lambda^{2}+2 p_{1} \lambda_{j}+q_{2} .
\end{gathered}
$$

Now we define the matrix $U$ by

$$
U=\left(\begin{array}{lllll}
u_{1} & u_{2} & u_{3} & \epsilon_{1} & u_{4}
\end{array}\right)=T K,
$$

where

$$
\epsilon_{1}=\frac{p_{0} p_{1}}{q_{1}^{2}}
$$

The matrix $K$ is given by

$$
K=d g\left(1,1,1, \epsilon_{1}\right)
$$

By (2.3) and (2.13), the transformation

$$
Y=U Z
$$

takes (2.1) into

$$
Z^{\prime}=\left(\Lambda-U^{-1} U^{\prime}\right) Z
$$

Now by (2.13),

$$
U^{-1} U^{\prime}=K^{-1} T^{-1} T^{\prime} K+K^{-1} K^{\prime},
$$

where

$$
K^{-1} K^{\prime}=d g\left(0,0,0, \epsilon_{1}^{-1} \epsilon_{1}^{\prime}\right),
$$

and we use (2.15).

Now if we write

$$
\begin{array}{ll}
U^{-1} U^{\prime}=\phi_{i j} & (1 \leq i, j \leq 4), \\
T^{-1} T^{\prime}=\psi_{i j} & (1 \leq i, j \leq 4),
\end{array}
$$


then by (2.18)-(2.21), we have

$$
\begin{gathered}
\phi_{i j}=\psi_{i j} \quad(1 \leq i, j \leq 3), \\
\phi_{44}=\psi_{44}+\epsilon_{1}^{-1} \epsilon_{1}^{\prime}, \\
\phi_{i 4}=\psi_{i 4} \epsilon_{1} \quad(1 \leq i \leq 3), \\
\phi_{4 j}=\epsilon_{1}^{-1} \psi_{4 j} \quad(1 \leq j \leq 3) .
\end{gathered}
$$

Now to work out $\phi_{i j}(1 \leq i, j \leq 4)$, it suffices to deal with $\psi_{i j}$ of the matrix $T^{-1} T^{\prime}$. Thus by (2.10), (2.12), (2.6), and (2.11), we obtain

$$
\psi_{i i}=\frac{1}{2} \frac{m_{i}^{\prime}}{m_{i}} \quad(1 \leq i \leq 4),
$$

and, for $i \neq j, 1 \leq i, j \leq 4$,

$$
\psi_{i j}=m_{i}^{-1}\left\{\lambda_{j}^{\prime}\left(p_{0} \lambda_{i}^{2}+\frac{1}{2} q_{1} \lambda_{i}\right)+\lambda_{i}\left(p_{0} \lambda_{j}^{2}+\frac{1}{2} q_{1} \lambda_{j}\right)^{\prime}+-\frac{1}{2} q_{2}^{\prime}-\left(p_{2} \lambda_{j}^{-1}\right)^{\prime}\right\} .
$$

Now we need to work out (2.23) and (2.24) in some detail in terms of $p_{0}, p_{1}, p_{2}, q_{1}$, and $q_{2}$, then (2.22) in order to determine the form of (2.17).

3. The matrices $\Lambda, T^{-1} T^{\prime}$, and $U^{-1} U^{\prime}$. In our analysis, we impose a basic condition on the coefficients as follows.

(I) $p_{i}(0 \leq i \leq 2)$ and $q_{i}(i=1,2)$ are nowhere zero in some interval $[a, \infty)$, and

$$
\begin{gathered}
\frac{p_{i}}{q_{i+1}}=o\left(\frac{q_{i+1}}{p_{i+1}}\right) \quad(i=0,1)(x \rightarrow \infty), \\
\frac{q_{1}}{p_{1}}=o\left(\frac{p_{1}}{q_{2}}\right) .
\end{gathered}
$$

If we write

$$
\epsilon_{1}=\frac{p_{0} p_{1}}{q_{1}^{2}}, \quad \epsilon_{2}=\frac{q_{1} q_{2}}{p_{1}^{2}}, \quad \epsilon_{3}=\frac{p_{2} p_{1}}{q_{2}^{2}},
$$

then by (3.1) for $(1 \leq i \leq 3)$,

$$
\epsilon_{i}=o(1) \quad(x \longrightarrow \infty)
$$

Now as in [1], we can solve the characteristic equation (2.4) asymptotically as $x \rightarrow \infty$. Using (3.1) and (3.2), we obtain the distinct eigenvalues $\lambda_{j}$ as

$$
\begin{aligned}
& \lambda_{1}=-\frac{p_{2}}{q_{2}}\left(1+\delta_{1}\right), \\
& \lambda_{2}=-\frac{q_{2}}{p_{1}}\left(1+\delta_{2}\right), \\
& \lambda_{3}=-\frac{p_{1}}{q_{1}}\left(1+\delta_{3}\right), \\
& \lambda_{4}=-\frac{q_{1}}{p_{0}}\left(1+\delta_{4}\right),
\end{aligned}
$$


where

$$
\delta_{1}=0\left(\epsilon_{3}\right), \quad \delta_{2}=0\left(\epsilon_{2}\right)+0\left(\epsilon_{3}\right), \quad \delta_{3}=0\left(\epsilon_{1}\right)+0\left(\epsilon_{2}\right), \quad \delta_{4}=0\left(\epsilon_{1}\right) .
$$

Now by (3.1), the ordering of $\lambda_{j}$ is such that

$$
\lambda_{j}=o\left(\lambda_{j+1}\right) \quad(x \rightarrow \infty, 1 \leq j \leq 3) .
$$

Now we work out $m_{j}(1 \leq j \leq 4)$ asymptotically as $x \rightarrow \infty$; hence by (3.2), (3.3), (3.4), (3.5), (3.6), (3.7), and (3.8), (2.12) gives, for $1 \leq j \leq 4$,

$$
\begin{aligned}
& m_{1}=q_{2}\left\{1+0\left(\epsilon_{3}\right)\right\}, \\
& m_{2}=-q_{2}\left\{1+0\left(\epsilon_{2}\right)+0\left(\epsilon_{3}\right)\right\}, \\
& m_{3}=\frac{p_{1}^{2}}{q_{1}}\left\{1+0\left(\epsilon_{1}\right)+0\left(\epsilon_{2}\right)\right\}, \\
& m_{4}=-\frac{q_{1}^{3}}{p_{0}^{2}}\left\{1+0\left(\epsilon_{1}\right)\right\} .
\end{aligned}
$$

Also by substituting $\lambda_{j}(j=1,2,3,4)$ into (2.12) and using (3.4), (3.5), (3.6), and (3.7), respectively, and differentiating, we obtain

$$
\begin{aligned}
& m_{1}^{\prime}=q_{2}^{\prime}\left\{1+0\left(\epsilon_{3}\right)\right\}+q_{2}\left\{0\left(\epsilon_{3}^{\prime}\right)+0\left(\epsilon_{3} \delta_{1}^{\prime}\right)+0\left(\epsilon_{2}^{\prime} \epsilon_{3}^{2}\right)+0\left(\epsilon_{2}^{\prime} \epsilon_{3}^{2} \epsilon_{3}^{3}\right)\right\}, \\
& m_{2}^{\prime}=-q_{2}^{\prime}\left\{1+0\left(\epsilon_{2}\right)+0\left(\epsilon_{3}\right)\right\}+q_{2}\left\{0\left(\delta_{2}^{\prime}\right)+0\left(\epsilon_{2}^{\prime}\right)+0\left(\epsilon_{1}^{\prime} \epsilon_{2}^{2}\right)\right\}, \\
& m_{3}^{\prime}=\left(\frac{p_{1}^{2}}{q_{1}}\right)^{\prime}\left\{1+0\left(\epsilon_{1}\right)+0\left(\epsilon_{2}\right)\right\}+\frac{p_{1}^{2}}{q_{1}}\left\{0\left(\delta_{3}^{\prime}\right)+0\left(\epsilon_{2}^{\prime}\right)+0\left(\epsilon_{1}^{\prime}\right)\right\}, \\
& m_{4}^{\prime}=-\left(\frac{q_{1}^{3}}{p_{0}^{2}}\right)^{\prime}\left\{1+0\left(\epsilon_{1}\right)\right\}+\frac{q_{1}^{3}}{p_{0}^{2}}\left\{0\left(\delta_{4}^{\prime}\right)+0\left(\epsilon_{2}^{\prime} \epsilon_{1}^{2}\right)+0\left(\epsilon_{1}^{\prime}\right)\right\} .
\end{aligned}
$$

At this stage we also require the following conditions.

(II)

$$
\frac{p_{0}^{\prime}}{p_{0}} \epsilon_{i}, \frac{p_{1}^{\prime}}{p_{1}} \epsilon_{i}, \frac{q_{1}^{\prime}}{q_{1}} \epsilon_{i}, \frac{q_{2}^{\prime}}{q_{2}} \epsilon_{i}, \frac{p_{2}^{\prime}}{p_{2}} \epsilon_{2}, \frac{p_{2}^{\prime}}{p_{2}} \epsilon_{3} \in L(a, x) \quad(1 \leq i \leq 3) .
$$

Further, differentiating (3.2) for $\epsilon_{i}(1 \leq i \leq 3)$, we obtain

$$
\begin{aligned}
& \epsilon_{1}^{\prime}=0\left(\frac{p_{0}^{\prime}}{p_{0}} \epsilon_{1}\right)+0\left(\frac{p_{1}^{\prime}}{p_{1}} \epsilon_{1}\right)+0\left(\frac{q_{2}}{q_{2}} \epsilon_{1}\right), \\
& \epsilon_{2}^{\prime}=0\left(\frac{q_{1}^{\prime}}{q_{1}} \epsilon_{2}\right)+0\left(\frac{q_{2}^{\prime}}{q_{2}} \epsilon_{2}\right)+0\left(\frac{p_{1}^{\prime}}{p_{1}} \epsilon_{2}\right), \\
& \epsilon_{3}^{\prime}=0\left(\frac{p_{2}^{\prime}}{p_{2}} \epsilon_{3}\right)+0\left(\frac{p_{1}^{\prime}}{p_{1}} \epsilon_{3}\right)+0\left(\frac{q_{2}^{\prime}}{q_{2}} \epsilon_{3}\right) .
\end{aligned}
$$


For reference, we note that by substituting (3.4), (3.5), (3.6), and (3.7) into (2.4) and differentiating, we obtain

$$
\begin{aligned}
& \delta_{1}^{\prime}=0\left(\epsilon_{3}^{\prime}\right)+0\left(\epsilon_{2}^{\prime} \epsilon_{3}^{2}\right)+0\left(\epsilon_{2}^{\prime} \epsilon_{3}^{3} \epsilon_{2}^{2}\right), \\
& \delta_{2}^{\prime}=0\left(\epsilon_{2}^{\prime}\right)+0\left(\epsilon_{3}^{\prime}\right)+0\left(\epsilon_{1}^{\prime} \epsilon_{3}^{2}\right), \\
& \delta_{3}^{\prime}=0\left(\epsilon_{1}^{\prime}\right)+0\left(\epsilon_{2}^{\prime}\right)+0\left(\epsilon_{3}^{\prime} \epsilon_{2}^{2}\right), \\
& \delta_{4}^{\prime}=0\left(\epsilon_{1}^{\prime}\right)+0\left(\epsilon_{2}^{\prime} \epsilon_{1}^{2}\right)+0\left(\epsilon_{3}^{\prime} \epsilon_{1}^{3} \epsilon_{2}^{2}\right) .
\end{aligned}
$$

Hence by (3.16) and (3.17), and (3.15),

$$
\epsilon_{j}^{\prime}, \delta_{j}^{\prime} \in L(a, \infty)
$$

For the diagonal elements $\psi_{i i}(1 \leq j \leq 4)$ in (2.23), we can now substitute the estimates (3.10), (3.11), (3.12), (3.13), and (3.14) into (2.23). We obtain

$$
\begin{aligned}
\psi_{11}= & \frac{1}{2} \frac{q_{2}^{\prime}}{q_{2}}+0\left(\frac{q_{2}^{\prime}}{q_{2}} \epsilon_{3}\right)+0\left(\epsilon_{3}^{\prime}\right)+0\left(\epsilon_{3} \delta_{1}^{\prime}\right)+0\left(\epsilon_{2}^{\prime} \epsilon_{3}^{2}\right)+0\left(\epsilon_{1}^{\prime} \epsilon_{2}^{2} \epsilon_{3}^{3}\right), \\
\psi_{22}= & \frac{1}{2} \frac{q_{2}^{\prime}}{q_{2}}+0\left(\frac{q_{2}^{\prime}}{q_{2}} \epsilon_{2}\right)+0\left(\frac{q_{2}^{\prime}}{q_{2}} \epsilon_{3}\right)+0\left(\delta_{2}^{\prime}\right)+0\left(\epsilon_{2}^{\prime}\right)+0\left(\epsilon_{2}^{\prime} \epsilon_{2}^{2}\right), \\
\psi_{33}= & \frac{1}{2}\left[2 \frac{p_{1}^{\prime}}{p_{1}}-\frac{q_{1}^{\prime}}{q_{1}}\right]+0\left(\frac{p_{1}^{\prime}}{p_{1}} \epsilon_{1}\right)+0\left(\frac{p_{1}^{\prime}}{p_{1}} \epsilon_{2}\right)+0\left(\frac{q_{1}^{\prime}}{q_{1}} \epsilon_{1}\right)+0\left(\frac{q_{1}^{\prime}}{q_{1}} \epsilon_{2}\right) \\
& +0\left(\delta_{3}^{\prime}\right)+0\left(\epsilon_{2}^{\prime}\right)+0\left(\epsilon_{1}^{\prime}\right), \\
\psi_{44}= & \frac{1}{2}\left[3 \frac{q_{1}^{\prime}}{q_{1}}-2 \frac{p_{0}^{\prime}}{p_{0}}\right]+0\left(\frac{q_{1}^{\prime}}{q_{1}} \epsilon_{1}\right)+0\left(\frac{p_{0}^{\prime}}{p_{0}} \epsilon_{1}\right)+0\left(\delta_{4}^{\prime}\right)+0\left(\epsilon_{2}^{\prime} \epsilon_{1}^{2}\right)+0\left(\epsilon_{1}^{\prime}\right) .
\end{aligned}
$$

Now for the nondiagonal elements $\psi_{i j}(i \neq j, 1 \leq i, j \leq 4)$, we consider (2.24). Hence (2.24) gives, for $i=1$ and $j=2$,

$$
\psi_{12}=m_{1}^{-1}\left\{\lambda_{2}^{\prime}\left(p_{0} \lambda_{1}^{2}+\frac{1}{2} q_{1} \lambda_{1}\right)+\lambda_{1}\left(p_{0} \lambda_{2}^{2}+\frac{1}{2} q_{1} \lambda_{2}\right)^{\prime}+-\frac{1}{2} q_{2}^{\prime}-\left(p_{2} \lambda_{2}^{-1}\right)^{\prime}\right\} .
$$

Now by (3.4), (3.5), (3.2), and (3.10), we have

$$
\begin{aligned}
m_{1}^{-1} \lambda_{2}^{\prime}\left(p_{0} \lambda_{1}^{2}+\frac{1}{2} q_{1} \lambda_{1}\right)= & \frac{1}{2}\left[2 \frac{q_{2}^{\prime}}{q_{2}}-\frac{p_{1}^{\prime}}{p_{1}}\right] \epsilon_{2} \epsilon_{3}\left\{1+0\left(\epsilon_{3}\right)\right\}+0\left(\epsilon_{2} \epsilon_{3} \delta_{2}^{\prime}\right), \\
m_{1}^{-1} \lambda_{1}\left(p_{0} \lambda_{1}^{2}+\frac{1}{2} q_{1} \lambda_{1}\right)^{\prime}= & 0\left(\epsilon_{2} \epsilon_{3} \delta_{2}^{\prime}\right)+0\left(\epsilon_{2}^{2} \epsilon_{1} \epsilon_{3}\right)+\left[\frac{p_{0}^{\prime}}{p_{0}}+2 \frac{q_{2}^{\prime}}{q_{2}}-2 \frac{p_{1}^{\prime}}{p_{1}}\right] \\
& +0\left(\epsilon_{2} \epsilon_{3}\right)\left[\frac{q_{1}^{\prime}}{q_{1}}+\frac{q_{2}^{\prime}}{q_{2}}-\frac{p_{1}^{\prime}}{p_{1}}\right], \\
-\frac{1}{2} q_{2}^{\prime} m_{1}^{-1}= & -\frac{1}{2} \frac{q_{2}^{\prime}}{q_{2}}+0\left(\frac{q_{2}^{\prime}}{q_{2}} \epsilon_{3}\right), \\
m_{1}^{-1}\left(p_{2} \lambda_{2}^{-1}\right)^{\prime}= & 0\left(\frac{p_{2}^{\prime}}{p_{2}} \epsilon_{3}\right)+0\left(\frac{p_{1}^{\prime}}{p_{1}} \epsilon_{3}\right)+0\left(\frac{q_{2}^{\prime}}{q_{2}} \epsilon_{3}\right)+0\left(\epsilon_{3} \delta_{2}^{\prime}\right) .
\end{aligned}
$$


Hence by (3.21), (3.22), (3.23), and (3.24), (3.20) gives

$$
\begin{aligned}
\psi_{12}= & -\frac{1}{2} \frac{q_{2}^{\prime}}{q_{2}}+0\left(\frac{q_{2}^{\prime}}{q_{2}} \epsilon_{3}\right)+0\left(\frac{p_{1}^{\prime}}{p_{1}} \epsilon_{3}\right)+0\left(\frac{p_{2}^{\prime}}{p_{2}} \epsilon_{3}\right)+0\left(\frac{p_{0}^{\prime}}{p_{0}} \epsilon_{1} \epsilon_{2}^{2} \epsilon_{3}\right) \\
& +0\left(\epsilon_{3} \delta_{2}^{\prime}\right)+0\left(\frac{q_{1}^{\prime}}{q_{1}} \epsilon_{2} \epsilon_{3}\right) .
\end{aligned}
$$

Similar work can be done for the other elements $\psi_{i j}$; so we obtain

$$
\begin{aligned}
& \psi_{13}=-\frac{1}{2} \frac{q_{2}^{\prime}}{q_{2}}+0\left(\frac{q_{2}^{\prime}}{q_{2}} \epsilon_{3}\right)+0\left(\frac{p_{1}^{\prime}}{p_{1}} \epsilon_{3}\right)+0\left(\frac{q_{1}^{\prime}}{q_{1}} \epsilon_{3}\right)+0\left(\epsilon_{3} \delta_{3}^{\prime}\right)+0\left(\frac{p_{0}^{\prime}}{p_{0}} \epsilon_{1} \epsilon_{3}\right)+0\left(\frac{p_{2}^{\prime}}{p_{2}} \epsilon_{2} \epsilon_{3}\right), \\
& \psi_{14}=-\frac{1}{2} \frac{q_{2}^{\prime}}{q_{2}}+0\left(\frac{q_{2}^{\prime}}{q_{2}} \epsilon_{3}\right)+0\left(\frac{q_{1}^{\prime}}{q_{1}} \epsilon_{1}^{-1} \epsilon_{3}\right)+0\left(\frac{p_{0}^{\prime}}{p_{0}} \epsilon_{1}^{-1} \epsilon_{3}\right)+0\left(\epsilon_{1}^{-1} \epsilon_{3} \delta_{4}^{\prime}\right) 0\left(\frac{p_{2}^{\prime}}{p_{2}} \epsilon_{1} \epsilon_{2} \epsilon_{3}\right), \\
& \psi_{21}=-\frac{1}{2} \frac{q_{2}^{\prime}}{q_{2}}+0\left(\frac{q_{2}^{\prime}}{q_{2}} \epsilon_{2}\right)+0\left(\frac{q_{2}^{\prime}}{q_{2}} \epsilon_{3}\right)+0\left(\delta_{1}^{\prime}\right)+0\left(\epsilon_{2} \frac{p_{2}^{\prime}}{p_{2}}\right)+0\left(\epsilon_{3} \frac{p_{2}^{\prime}}{p_{2}}\right) \\
& +0\left(\frac{q_{1}^{\prime}}{q_{1}} \epsilon_{2} \epsilon_{3}\right)+0\left(\frac{p_{0}^{\prime}}{p_{0}} \epsilon_{1} \epsilon_{2}^{2} \epsilon_{3}^{2}\right) \text {, } \\
& \psi_{23}=\left[\frac{1}{2} \frac{q_{1}^{\prime}}{q_{1}}-\frac{p_{1}^{\prime}}{p_{1}}+\frac{1}{2} \frac{q_{2}^{\prime}}{q_{2}}\right]+0\left(\frac{q_{1}^{\prime}}{q_{1}} \epsilon_{1}\right)+0\left(\frac{q_{1}^{\prime}}{q_{1}} \epsilon_{2}\right)+0\left(\frac{q_{1}^{\prime}}{q_{1}} \epsilon_{3}\right)+0\left(\frac{p_{1}^{\prime}}{p_{1}} \epsilon_{1}\right) \\
& +0\left(\frac{p_{1}^{\prime}}{p_{1}} \epsilon_{2}\right)+0\left(\frac{p_{1}^{\prime}}{p_{1}} \epsilon_{3}\right)+0\left(\frac{q_{2}^{\prime}}{q_{2}} \epsilon_{2}\right)+0\left(\frac{q_{2}^{\prime}}{q_{2}} \epsilon_{3}\right)+0\left(\delta_{3}^{\prime}\right)+0\left(\frac{p_{0}^{\prime}}{p_{0}} \epsilon_{1}\right)+0\left(\epsilon_{2} \epsilon_{3} \frac{p_{2}^{\prime}}{p_{2}}\right) \text {, } \\
& \psi_{24}=\epsilon_{1}^{-1}\left[\frac{1}{2} \frac{q_{1}^{\prime}}{q_{1}}+0\left(\frac{q_{1}^{\prime}}{q_{1}} \epsilon_{1}\right)+0\left(\frac{q_{1}^{\prime}}{q_{1}} \epsilon_{2}\right)+0\left(\frac{q_{1}^{\prime}}{q_{1}} \epsilon_{3}\right)+0\left(\frac{p_{0}^{\prime}}{p_{0}} \epsilon_{1}\right)+0\left(\frac{p_{0}^{\prime}}{p_{0}} \epsilon_{2}\right)\right. \\
& \left.+0\left(\frac{p_{0}^{\prime}}{p_{0}} \epsilon_{3}\right)+0\left(\delta_{4}^{\prime}\right)+0\left(\frac{q_{2}^{\prime}}{q_{2}} \epsilon_{1}\right)+0\left(\frac{p_{2}^{\prime}}{p_{2}} \epsilon_{1}^{2} \epsilon_{2} \epsilon_{3}\right)\right] \text {, } \\
& \psi_{31}=0\left(\frac{p_{2}^{\prime}}{p_{2}} \epsilon_{2}\right)+0\left(\frac{q_{2}^{\prime}}{q_{2}} \epsilon_{2}\right)+0\left(\delta_{1}^{\prime} \epsilon_{2}\right)+0\left(\frac{q_{1}^{\prime}}{q_{1}} \epsilon_{2} \epsilon_{3}\right)+0\left(\frac{p_{0}^{\prime}}{p_{0}} \epsilon_{1} \epsilon_{2}^{2} \epsilon_{3}^{2}\right), \\
& \psi_{32}=0\left(\frac{q_{2}^{\prime}}{q_{2}} \epsilon_{2}\right)+0\left(\frac{p_{1}^{\prime}}{p_{1}} \epsilon_{2}\right)+0\left(\epsilon_{2} \delta_{2}^{\prime}\right)+0\left(\epsilon_{1} \epsilon_{2}^{2} \frac{p_{0}^{\prime}}{p_{0}}\right)+0\left(\frac{q_{1}^{\prime}}{q_{1}} \epsilon_{2}\right)+0\left(\epsilon_{2} \epsilon_{3} \frac{p_{2}^{\prime}}{p_{2}}\right) \text {, } \\
& \psi_{34}=\epsilon_{1}^{-1}\left[-\frac{1}{2} \frac{q_{1}^{\prime}}{q_{1}}+0\left(\frac{q_{1}^{\prime}}{q_{1}} \epsilon_{1}\right)+0\left(\frac{q_{1}^{\prime}}{q_{1}} \epsilon_{2}\right)+0\left(\frac{p_{0}^{\prime}}{p_{0}} \epsilon_{1}\right)+0\left(\frac{p_{0}^{\prime}}{p_{0}} \epsilon_{2}\right)\right. \\
& \left.+0\left(\delta_{4}^{\prime}\right)+0\left(\frac{q_{1}^{\prime}}{q_{1}} \epsilon_{1} \epsilon_{2}\right)+0\left(\frac{p_{2}^{\prime}}{p_{2}} \epsilon_{1}^{2} \epsilon_{2}^{2} \epsilon_{3}\right)\right], \\
& \psi_{41}=\epsilon_{1}\left[0\left(\frac{q_{1}^{\prime}}{q_{1}} \epsilon_{2} \epsilon_{3}\right)+0\left(\frac{q_{2}^{\prime}}{q_{2}} \epsilon_{1} \epsilon_{2}\right)+0\left(\frac{p_{2}^{\prime}}{p_{2}} \epsilon_{1} \epsilon_{2}\right)+0\left(\delta_{1}^{\prime} \epsilon_{1} \epsilon_{2}\right)+0\left(\frac{p_{0}^{\prime}}{p_{0}} \epsilon_{1} \epsilon_{2}^{2} \epsilon_{3}^{2}\right)\right], \\
& \psi_{42}=0\left(\frac{q_{2}^{\prime}}{q_{2}} \epsilon_{1} \epsilon_{2}\right)+0\left(\frac{p_{0}^{\prime}}{p_{0}} \epsilon_{1} \epsilon_{2}\right)+0\left(\delta_{2}^{\prime} \epsilon_{1} \epsilon_{2}\right)+0\left(\frac{q_{1}^{\prime}}{q_{1}} \epsilon_{1}^{2} \epsilon_{2}^{2}\right)+0\left(\frac{p_{0}^{\prime}}{p_{0}} \epsilon_{1}^{2} \epsilon_{2}^{2}\right)+0\left(\frac{p_{2}^{\prime}}{p_{2}} \epsilon_{1}^{2} \epsilon_{2} \epsilon_{3}\right), \\
& \psi_{43}=\epsilon_{1}\left[-\frac{1}{2} \frac{q_{1}^{\prime}}{q_{1}}+0\left(\frac{p_{1}^{\prime}}{p_{1}} \epsilon_{1}\right)+0\left(\frac{q_{1}^{\prime}}{q_{1}} \epsilon_{1}\right)+0\left(\frac{q_{1}^{\prime}}{q_{1}} \epsilon_{2}\right)+0\left(\delta_{3}^{\prime} \epsilon_{1}\right)+0\left(\frac{p_{0}^{\prime}}{p_{0}} \epsilon_{1}\right)\right. \\
& \left.+0\left(\frac{p_{2}^{\prime}}{p_{2}} \epsilon_{1} \epsilon_{2}^{2} \epsilon_{3}\right)+0\left(\frac{q_{2}^{\prime}}{q_{2}} \epsilon_{1} \epsilon_{2}\right)\right] \text {. }
\end{aligned}
$$


Now we need to work out (2.22) in order to determine the form (2.17). Now by (3.19), and (3.25) and (3.26), (2.22) will give

$$
\begin{aligned}
& \phi_{11}=\frac{1}{2} \frac{q_{2}^{\prime}}{q_{2}}+0\left(\Delta_{1}\right), \quad \phi_{22}=\frac{1}{2} \frac{q_{2}^{\prime}}{q_{2}}+0\left(\Delta_{2}\right), \\
& \phi_{33}=\frac{p_{1}^{\prime}}{p_{1}}-\frac{1}{2} \frac{q_{1}^{\prime}}{q_{1}}+0\left(\Delta_{3}\right), \quad \phi_{44}=\frac{p_{1}^{\prime}}{p_{1}}-\frac{1}{2} \frac{q_{1}^{\prime}}{q_{1}}+0\left(\Delta_{4}\right), \\
& \phi_{12}=\frac{1}{2} \frac{q_{2}^{\prime}}{q_{2}}+0\left(\Delta_{5}\right), \quad \phi_{13}=-\frac{1}{2} \frac{q_{2}^{\prime}}{q_{2}}+0\left(\Delta_{6}\right), \\
& \phi_{14}=0\left(\Delta_{7}\right), \quad \phi_{21}=-\frac{1}{2} \frac{q_{2}^{\prime}}{q_{2}}+0\left(\Delta_{8}\right), \\
& \phi_{23}=\frac{1}{2}\left(\frac{q_{1}^{\prime}}{q_{1}}+\frac{q_{2}^{\prime}}{q_{2}}\right)-\frac{p_{1}^{\prime}}{p_{1}}+0\left(\Delta_{9}\right), \quad \phi_{24}=\frac{1}{2} \frac{q_{1}^{\prime}}{q_{1}}+0\left(\Delta_{10}\right), \\
& \phi_{31}=0\left(\Delta_{11}\right), \quad \phi_{32}=0\left(\Delta_{12}\right), \quad \phi_{34}=-\frac{1}{2} \frac{q_{1}^{\prime}}{q_{1}}+0\left(\Delta_{13}\right), \\
& \phi_{41}=0\left(\Delta_{14}\right), \quad \phi_{42}=0\left(\Delta_{15}\right), \quad \phi_{43}=-\frac{1}{2} \frac{q_{1}^{\prime}}{q_{1}}+0\left(\Delta_{16}\right),
\end{aligned}
$$

where

$$
\Delta_{i} \in L(a, \infty) \quad(1 \leq i \leq 16)
$$

by (3.15) and (3.18).

Now by (3.27) and (3.28), we write the system (2.17) as

$$
Z^{\prime}=(\Lambda+R+S) Z
$$

where

$$
R=\left[\begin{array}{cccc}
-\eta_{1} & \eta_{1} & \eta_{1} & 0 \\
\eta_{1} & -\eta_{1} & \eta_{2}-\eta_{1} & -\eta_{3} \\
0 & 0 & -\eta_{2} & \eta_{3} \\
0 & 0 & \eta_{3} & -\eta_{2}
\end{array}\right]
$$

with

$$
\eta_{1}=\frac{1}{2} \frac{q_{2}^{\prime}}{q_{2}}, \quad \eta_{2}=\frac{\left(p_{1} q_{1}^{-1 / 2}\right)^{\prime}}{p_{1} q_{1}^{-1 / 2}}, \quad \eta_{3}=\frac{1}{2} \frac{q_{1}^{\prime}}{q_{1}},
$$

and $S \in L(a, \infty)$ by (3.28).

4. The Euler case. Now we deal with (1.3) more generally, so we write (1.3) as

$$
\eta_{k}=\sigma_{k} \frac{q_{1}}{p_{0}}\left(1+\varphi_{k}\right) \quad(1 \leq k \leq 3),
$$

where $\sigma_{k}(1 \leq k \leq 3)$ are nonzero constants, $\varphi_{k}(x) \rightarrow 0(1 \leq k \leq 3, x \rightarrow \infty)$, and also at this stage we let

$$
\varphi_{k}^{\prime} \in L(a, \infty) \quad(1 \leq k \leq 3)
$$


We note that by (4.1), the matrix $\Lambda$ no longer dominates $R$ and so Eastham's theorem [6, Section 2] is not satisfied which means that we have to carry out a second diagonalization of the system (3.29).

First we write

$$
\Lambda+R=\lambda_{4}\left\{S_{1}+S_{2}\right\}
$$

and we need to work out the matrix $S_{1}=$ const with the matrix $S_{2}(x)=o(1)$ as $x \rightarrow \infty$ using (3.4), (3.5), (3.6), and (3.7) and the Euler case (4.1). Hence after some calculations, we obtain

$$
\begin{aligned}
S_{1} & =\left(\begin{array}{cccc}
\sigma_{1} & -\sigma_{1} & -\sigma_{1} & 0 \\
-\sigma_{1} & \sigma_{1} & \sigma_{1}-\sigma_{2} & \sigma_{3} \\
0 & 0 & \sigma_{2} & -\sigma_{3} \\
0 & 0 & -\sigma_{3} & \sigma_{2}
\end{array}\right), \\
S_{2}(x) & =\left(\begin{array}{cccc}
u_{1} & u_{2} & u_{2} & 0 \\
u_{2} & u_{3} & u_{4} & u_{5} \\
0 & 0 & u_{6} & -u_{5} \\
0 & 0 & -u_{5} & -u_{7}
\end{array}\right),
\end{aligned}
$$

where

$$
\begin{gathered}
u_{1}=\lambda_{1} \lambda_{4}^{-1}-u_{2}, \quad u_{2}=-\sigma_{1}\left(\varphi_{1}-\delta_{4}\right)\left(1+\delta_{4}\right)^{-1}, \\
u_{3}=\lambda_{2} \lambda_{4}^{-1}-u_{2}, \quad u_{4}=-u_{2}+u_{7}, \\
u_{5}=\sigma_{3}\left(\varphi_{3}-\delta_{4}\right)\left(1+\delta_{4}\right)^{-1}, \quad u_{6}=\lambda_{3} \lambda_{4}^{-1}-u_{7}, \\
u_{7}=-\sigma_{2}\left(\varphi_{2}-\delta_{4}\right)\left(1+\delta_{4}\right)^{-1} .
\end{gathered}
$$

It is clear that by (3.9) and (3.8), $S_{2}(x) \rightarrow 0$ as $x \rightarrow \infty$. Hence we diagonalize the constant matrix $S_{1}$. Now the eigenvalues of the matrix $S_{1}$ are given by

$$
\alpha_{1}=0, \quad \alpha_{2}=2 \sigma_{1}, \quad \alpha_{3}=\sigma_{2}+\sigma_{3}, \quad \alpha_{4}=\sigma_{2}-\sigma_{3} .
$$

Let

$$
\sigma_{2} \neq\left( \pm \sigma_{3}, \pm \sigma_{3}+2 \sigma_{1}\right)
$$

Hence by (4.7), the eigenvalues $\alpha_{i}(1 \leq i \leq 4)$ are distinct. Thus we use the transformation

$$
Z=T_{1} W
$$

in (3.29), where $T_{1}$ diagonalizes the constant matrix $S_{1}$. Then (3.29) transforms to

$$
W^{\prime}=\left(\Lambda_{1}+M+T_{1}^{-1} S T_{1}\right) W
$$


where

$$
\begin{gathered}
\Lambda_{1}=\lambda_{4} T_{1}^{-1} S_{1} T_{1}=\operatorname{diag}\left(v_{1}, v_{2}, v_{3}, v_{4}\right)=\lambda_{4} \operatorname{diag}\left(\alpha_{1}, \alpha_{2}, \alpha_{3}, \alpha_{4}\right), \\
M=\lambda_{4} T_{1}^{-1} S_{2} T_{1}, \\
T_{1}^{-1} S T_{1} \in L(a, \infty) .
\end{gathered}
$$

Now we can apply the asymptotic theorem of Eastham in [6, Section 2] to (4.9) provided only that $\Lambda_{1}$ and $M$ satisfy the conditions in [6, Section 2].

We first require that the $v_{j}(1 \leq j \leq 4)$ are distinct, and this holds because the $\alpha_{j}$ $(1 \leq j \leq 4)$ are distinct.

Second, we need to show that

$$
\frac{M}{u_{i}-v_{j}} \longrightarrow 0 \quad(x \rightarrow \infty)
$$

for $i \neq j$ and $1 \leq i, j \leq 4$. Now

$$
\frac{M}{v_{i}-v_{j}}=\left(\alpha_{i}-\alpha_{j}\right)^{-1} T_{1}^{-1} S_{2} T_{1}=o(1) \quad(x \longrightarrow \infty) .
$$

Thus (4.11) holds. Third, we need to show that

$$
S_{2}^{\prime} \in L(a, \infty)
$$

Thus it suffices to show that

$$
u_{i}^{\prime}(x) \in L(a, \infty) \quad(1 \leq i \leq 8)
$$

Now, by (3.4), (3.5), (3.6), (3.7), and (4.5),

$$
\begin{gathered}
u_{1}^{\prime}=0\left(\epsilon_{1}^{\prime} \epsilon_{2} \epsilon_{3}\right)+0\left(\epsilon_{2}^{\prime} \epsilon_{1} \epsilon_{3}\right)+0\left(\epsilon_{3}^{\prime} \epsilon_{1} \epsilon_{2}\right)+0\left(\varphi^{\prime}\right)+0\left(\delta_{4}^{\prime}\right), \\
u_{2}^{\prime}=0\left(\varphi^{\prime}\right)+0\left(\delta_{4}^{\prime}\right), \\
u_{3}^{\prime}=0\left(\epsilon_{1}^{\prime} \epsilon_{2}\right)+0\left(\epsilon_{2}^{\prime} \epsilon_{1}\right)+0\left(\delta_{2}^{\prime} \epsilon_{1} \epsilon_{2}\right)+0\left(\varphi_{1}^{\prime}\right)+0\left(\delta_{4}^{\prime}\right), \\
u_{4}^{\prime}=0\left(\varphi_{1}^{\prime}\right)+0\left(\delta_{4}^{\prime}\right)+0\left(\varphi_{2}^{\prime}\right) \\
u_{5}^{\prime}=0\left(\varphi_{3}^{\prime}\right)+0\left(\delta_{4}^{\prime}\right) \\
u_{6}^{\prime}=0\left(\epsilon_{1}^{\prime}\right)+0\left(\epsilon_{1} \delta_{3}^{\prime}\right)+0\left(\varphi_{2}^{\prime}\right)+0\left(\delta_{4}^{\prime}\right), \\
u_{7}^{\prime}=0\left(\varphi_{2}^{\prime}\right)+0\left(\delta_{4}^{\prime}\right) .
\end{gathered}
$$

Thus by (4.15), (3.18), and (4.2), (4.14) holds and consequently (4.13) holds. Now we state our main theorem for (1.1).

THEOREM 4.1. Let the coefficients $q_{1}, q_{2}$, and $p_{1}$ in (1.1) be in $\mathrm{C}^{(2)}[a, \infty)$ and let $p_{0}$ and $p_{2}$ be $\mathrm{C}^{(1)}[a, \infty)$. Let (3.1), (3.15), (4.1), (4.2), and (4.7) hold.

Let

$$
\begin{gathered}
\operatorname{Re} I_{j}(x) \quad(j=1,2), \\
\operatorname{Re}\left[\lambda_{1}+\lambda_{2}-\lambda_{3}-\lambda_{4}-2 \eta_{1}+2 \eta_{2} \pm I_{1} \pm I_{2}\right]
\end{gathered}
$$


be one sign in $[a, \infty)$, where

$$
\begin{aligned}
& I_{1}=\left[4 \eta_{1}^{2}+\left(\lambda_{1}-\lambda_{2}\right)^{2}\right]^{1 / 2}, \\
& I_{2}=\left[4 \eta_{3}^{2}+\left(\lambda_{3}-\lambda_{4}\right)^{2}\right]^{1 / 2} .
\end{aligned}
$$

Then (1.1) has solutions

$$
\begin{gathered}
y_{1} \sim q_{2}^{-1 / 2} \exp \left(\frac{1}{2} \int_{a}^{x}\left[\lambda_{1}+\lambda_{2}+I_{1}\right] d t\right), \\
y_{2}=o\left\{q_{1}^{1 / 2} \exp \left(\frac{1}{2} \int_{a}^{x}\left[\lambda_{1}+\lambda_{2}-I_{1}\right] d t\right)\right\}, \\
y_{k}=o\left\{q_{1}^{-1 / 2} p_{1}^{-1} \exp \left(\frac{1}{2} \int_{a}^{x}\left[\lambda_{3}+\lambda_{4}+(-1)^{k+1} I_{2}\right] d t\right)\right\} \quad(k=3,4) .
\end{gathered}
$$

Proof. Before applying the theorem in [6, Section 2], we show that the eigenvalues $\mu_{k}$ of $\Lambda_{1}+M$ satisfy the dichotomy condition [8]. As in [1], the dichotomy condition holds if

$$
\left(\mu_{j}-\mu_{k}\right)=f+g \quad(j \neq k, 1 \leq j, k \leq 4),
$$

where $f$ has one sign in $[a, \infty)$ and $g$ belongs to $L[a, \infty)[6,(1.5)]$. Now since the eigenvalues of $\Lambda_{1}+M$ are the same as the eigenvalues of $\Lambda+R$, hence by (2.3) and (3.23),

$$
\begin{aligned}
& \mu_{k}=\frac{1}{2}\left(\lambda_{3}+\lambda_{2}-2 \eta_{1}\right)+\frac{1}{2}(-1)^{k+1} I_{1} \quad(k=1,2), \\
& \mu_{k}=\frac{1}{2}\left(\lambda_{3}+\lambda_{4}-2 \eta_{2}\right)+\frac{(-1)^{k+1}}{2} I_{2} \quad(k=3,4) .
\end{aligned}
$$

Thus by (4.20) and (4.16), (4.19) holds. Since (4.9) satisfies all the conditions for the asymptotic result [6, Section 2], it follows that, as $x \rightarrow \infty$, (4.9) has four linearly independent solutions

$$
W_{k}(x)=\left\{e_{k}+o(1)\right\} \exp \left(\int_{a}^{x} \mu_{k}(t) d t\right)
$$

with $e_{k}$ being the coordinate vector with $k$ th component unity and other components being zero. Now we transform back to $Y$ by means of (2.16) and (4.8), where $T_{1}$ in (4.8) is given by

$$
T_{1}=\left(\begin{array}{cccc}
1 & 1 & 0 & 0 \\
1 & -1 & -1 & -1 \\
0 & 0 & 1 & 1 \\
0 & 0 & -1 & 1
\end{array}\right)
$$

We obtain

$$
Y_{k}(x)=U T_{1} W_{k}(x) \quad(1 \leq k \leq 4) .
$$

Now using (2.13), (2.14), (2.15), (4.20), (4.21), (4.22), and (3.31) in (4.23) and carrying out the integration of $(1 / 2)\left(q_{2}^{\prime} / q\right)$ and $\left(q_{1}^{1 / 2} p_{1}^{-1}\right)^{\prime} / q_{1}^{1 / 2} p_{1}^{-1}$, for $1 \leq k \leq 4$, we obtain (4.18). 
5. Discussion. (i) In the familiar case, the coefficients which are covered by Theorem 4.1 are $p_{i}(x)=c_{i} x^{\alpha_{j}}(i=0,1,2)$ and $q_{i}(x)=c_{i+2} x^{\alpha_{i+2}}(i=1,2)$ with real constants $\alpha_{i}$ and $c_{i}(0 \leq i \leq 4)$. Then the Euler case (4.2) is given by

$$
\alpha_{0}-\alpha_{3}=1
$$

The values of $\sigma_{k}(1 \leq k \leq 3)$ in (4.1) are given by

$$
\sigma_{1}=\frac{1}{2} \alpha_{4} c_{0} c_{3}^{-1}, \quad \sigma_{2}=\left(\alpha_{1}-\frac{1}{2} \alpha_{3}\right) c_{0} c_{3}^{-1}, \quad \sigma_{3}=\frac{1}{2} \alpha_{3} c_{0} c_{3}^{-1} .
$$

Also in this example, $\varphi_{k}(x)=0$ in (4.1).

(ii) Also the theorem covered the class of the coefficients

$$
\begin{gathered}
p_{0}=c_{0} x^{\alpha_{0}} e^{x^{b}}, \quad p_{1}=c_{1} x^{\alpha_{1}} e^{(1 / 4) x^{b}}, \quad p_{2}=c_{2} x^{\alpha_{2}} e^{-3 x^{b}}, \\
q_{1}=c_{3} x^{\alpha_{3}} e^{x^{b}}, \quad q_{2}=c_{4} x^{\alpha_{4}} e^{-x^{b}}
\end{gathered}
$$

with real constants $c_{i}, \alpha_{i}(0 \leq i \leq 4)$ and $b(>0)$.

The Euler case (4.1) is given by

$$
\alpha_{3}-\alpha_{0}=b-1
$$

The values of $\sigma_{k}(1 \leq k \leq 4)$ in (4.1) are given by

$$
\sigma_{1}=\frac{1}{2} b c_{0} c_{3}^{-1}, \quad \sigma_{2}=\frac{1}{2} \sigma_{1}, \quad \sigma_{3}=-\sigma_{1} .
$$

Also

$$
\varphi_{1}=-\alpha_{4} b^{-1} x^{-b}, \quad \varphi_{2}=4 b^{-1}\left(\frac{1}{2} \alpha_{3}-\alpha_{1}\right) x^{-b}, \quad \varphi_{3}=b^{-1} \alpha_{3} x^{-b} .
$$

\section{REFERENCES}

[1] A. S. A. Al-Hammadi, Asymptotic theory for a class of fourth-order differential equations, Mathematika 43 (1996), no. 1, 198-208.

[2] _ Asymptotic formulae of Liouville-Green type for a general fourth-order differential equation, Rocky Mountain J. Math. 28 (1998), no. 3, 801-812.

[3] A. S. A. Al-Hammadi and M. S. P. Eastham, Higher-order differential equations with small oscillatory coefficients, J. London Math. Soc. (2) 40 (1989), no. 3, 507-518.

[4] M. S. P. Eastham, Asymptotic theory for a critical class of fourth-order differential equations, Proc. Roy. Soc. London Ser. A 383 (1982), no. 1785, 465-476.

[5] - On the eigenvectors for a class of matrices arising from quasiderivatives, Proc. Roy. Soc. Edinburgh Sect. A 97 (1984), 73-78.

[6] _ . The asymptotic solutions of linear differential systems, Mathematika 32 (1985), no. 1, 131-138.

[7] W. N. Everitt and A. Zettl, Generalized symmetric ordinary differential expressions. I. The general theory, Nieuw Arch. Wisk. (3) 27 (1979), no. 3, 363-397.

[8] N. Levinson, The asymptotic nature of solutions of linear systems of differential equations, Duke Math. J. 15 (1948), 111-126. 
[9] P. W. Walker, Asymptotica of the solutions to $\left[\left(r y^{\prime \prime}\right)^{\prime}-p y^{\prime}\right]^{\prime}+q y=\sigma y$, J. Differential Equations 9 (1971), 108-132.

[10] _ Asymptotics for a class of fourth order differential equations, J. Differential Equations 11 (1972), 321-334.

A. S. A. Al-Hammadi: Department of Mathematics, College of Science, University of Bahrain, P.O. Box 32088, Bahrain

E-mail address: aa7hammadi@sci.uob.bh 


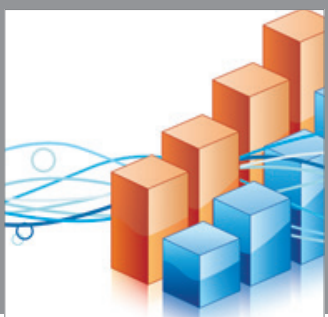

Advances in

Operations Research

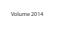

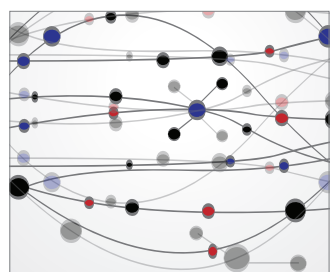

\section{The Scientific} World Journal
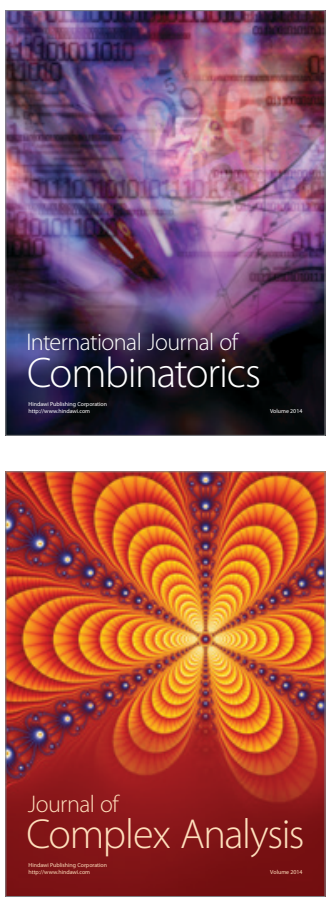

International Journal of

Mathematics and

Mathematical

Sciences
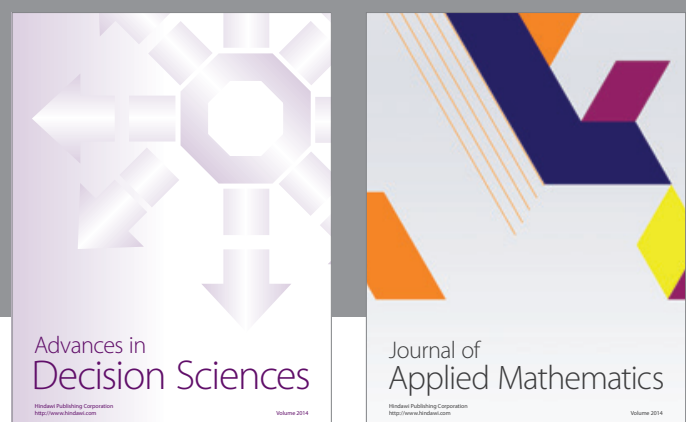

Journal of

Applied Mathematics
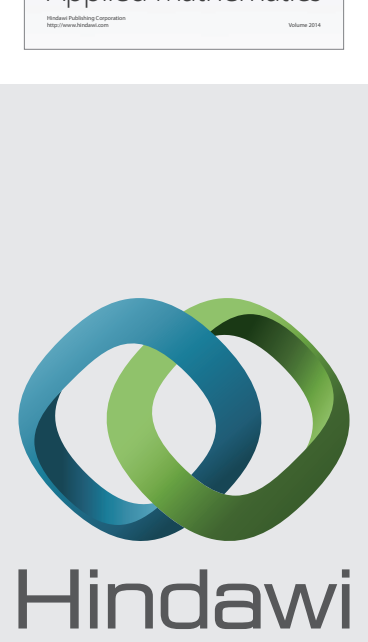

Submit your manuscripts at http://www.hindawi.com
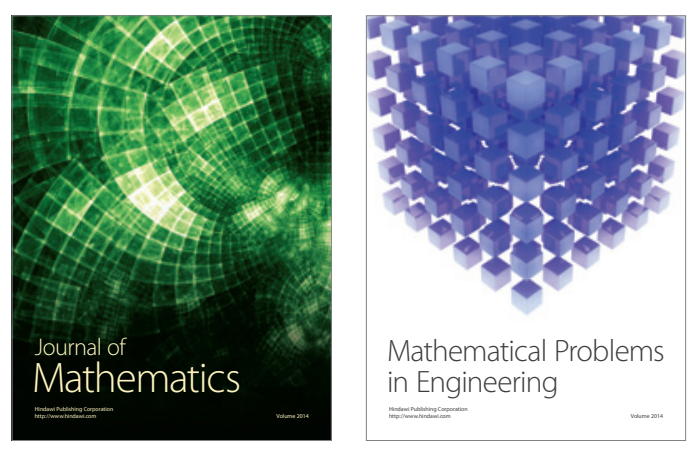

Mathematical Problems in Engineering
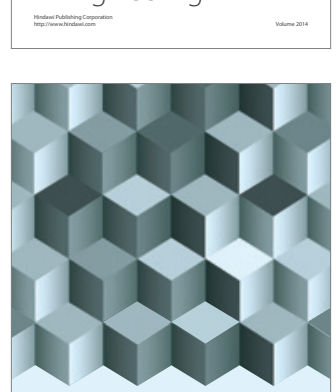

Journal of

Function Spaces
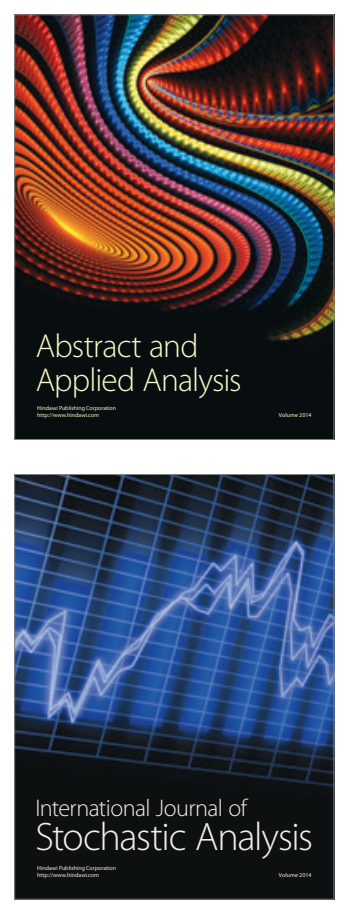

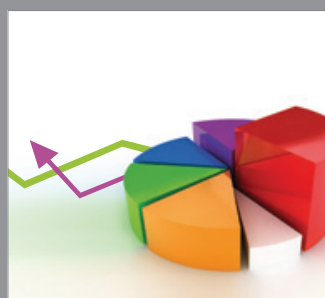

ournal of

Probability and Statistics

Promensencen
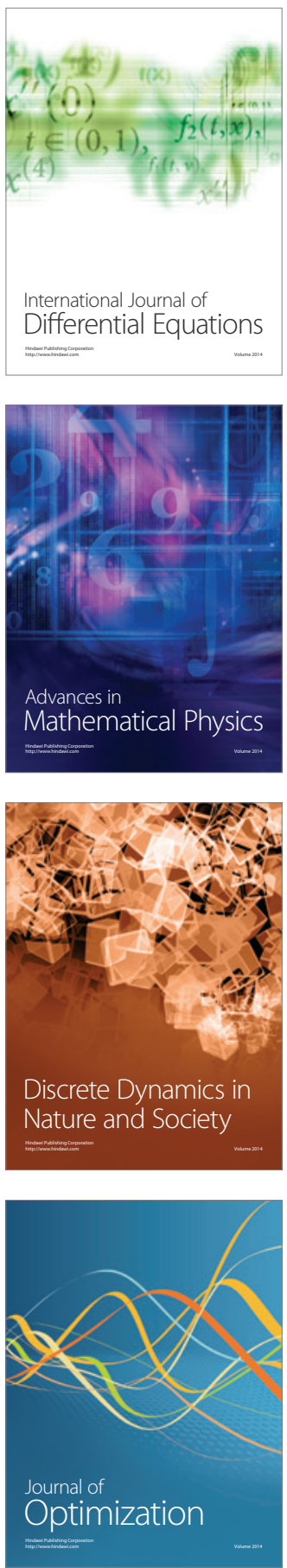